\title{
Wideband Voltage Controlled Oscillators for Cognitive Radio Systems
}

\author{
Alessandro Acampora and Apostolos Georgiadis \\ Centre Tecnologic de Telecomunicacions de Catalunya (CTTC)
}

Spain

\section{Introduction}

In the latest years much research effort was devoted to envision a new paradigm for wireless transmission. Results from recent works (Wireless Word Research Forum, 2005) indicate that a possible solution would lie in utilizing in a more efficient manner the diverse Radio Access Technologies ${ }^{1}$ (RATs) that are available nowadays, with the purpose of enabling interoperability among them and convergence into one global telecom infrastructure (beyond 3G).

Turning such a representation into reality requires endowing both the network and the user terminal with advanced management functionalities to ensure an effective utilization of radio resources. From the network providers' side, this translates in devising support for heterogeneous RATs, to map or reallocate traffic stream according to QoS requirements², while from the users terminals' side a major step towards a smarter utilization of radio resources consists in enabling reconfigurability, so to adapt dynamically the transmission to the spectrum environment in such a way that is no longer required to have fixed frequency bands mapped uniquely to specific RAT. Through a smarter selection of unused frequency bands spanning various access technologies, is possible to achieve the maximization of each RAT capacity both in time and space (within a geographical area) while at the same time minimizing the mutual interference. The support for heterogeneous access technologies on the network side and reconfigurable devices on the terminal side constitutes the essence of the Cognitive Radio paradigm (Akyildiz et al., 2006).

Several spectrum management protocols have been proposed from different research bodies/agencies worldwide, e.g. DARPA XG OSA "Open Spectrum Access" in (Akyildiz et al., 2006). However, all of them pose relevant challenges from the hardware implementation point of view to achieve adaptive utilization of radio resources. In fact, in order to identify unused portion of the spectrum at a specific time in a certain geographical area is necessary

\footnotetext{
${ }^{1}$ Consider for example GSM/GPRS for 2G cellular network, UMTS (HSPA) for 3G (3.5G) cellular network delivering high speed data transmission and nomadic internet access, WLAN for wireless local area networks, WIMAX for providing wireless metropolitan internet access.

${ }^{2}$ Examples of protocols offering support for managing heterogeneous networks are GAN "Generic Access Network" and ANDSF "Access Network Discovery and Selection Function", details can be found in (Ferrus et al., 2010; Frei et al., 2011)
} 
to execute a real-time, wide-band sensing, capable of spanning across the frequency bands of the various RATs. To that aim the frequency of the local oscillator in the transceiver module of a user terminal should be continuously swept across a wide frequency range, thus motivating the need for wideband tunable oscillators as an enabling technology for successful deployment of Cognitive Radio capabilities. There are many possibilities to implement an oscillator with a variable frequency, the most common of which is referred to Voltage Controlled Oscillator (VCO) in which generally altering a DC voltage at a convenient node in the circuit produces a frequency shifting in the sinusoidal output waveform. In the case of VCOs derived by harmonic oscillators ${ }^{3}$ this could be due the variation in the parameters of the nonlinear device model (Sun, 1972), or simply the effect of an added varactor in the embedded fixed frequency oscillator network (Cohen, 1979; Peterson, 1980) so that the phase of the signal across the feedback path could be varied, and the its frequency adjusted as a result of a variable capacitive loading.

A suitable VCO for Cognitive Radio applications should provide large tuning bandwidths in order to cover the spectrum of the diverse RATs, and has to cope with additional limitations due to space occupancy of the circuit (the possibility of having an integrated chip), its spectral purity (expressed in terms of low phase noise), the linearity of the tuning function, its harmonic rejection (related to the content of higher order harmonics with respect to the fundamental) its output power (which is assumed to be as high as possible) its efficiency (the amount of RF energy output produced relative to the DC power supply) and DC current consumption (which ideally should be kept low). Meeting all these requirements might be made easier if instead of using conventional circuit techniques, one considers microwave distributed voltage controlled oscillators (DVCO) (Divina \& Škvor 1998; Wu \& Hajimiri, 2000; Yuen \& Tsang, 2004).

Essentially a distributed oscillator consists of a distributed amplifier (Škvor et al., 1992; Wong, 1993) in which a feedback path is created in order to build up and sustain oscillations. In order to vary the oscillation frequency in a prescribed range is possible to introduce a varactor in the feedback loop (Yuen \& Tsang, 2004) or use some advanced techniques like the "current steering" in (Wu \& Hajimiri, 2000). However, these solutions do not provide a real wide-band operation since relative tuning ranges of nearly $12 \%$ are attained both in (Yuen \& Tsang, 2004) and in (Wu \& Hajimiri, 2000). Instead the reverse mode DVCO working principle (Divina \& Škvor, 1998; Škvor et al., 1992) based upon a feedback path for backward scattered waves in the drain line (hence the name) and the concurrent variation the active devices' gate voltages as a mean for adjusting the oscillation frequency, presents a wide tuning range, up to a frequency decade (Škvor et al., 1992) a good output power, on the order of $+10 \mathrm{dBm}$, adequate suppression of higher harmonics with typical values for second and third order harmonic rejection of $-20 \mathrm{dBc},-30 \mathrm{dBc}$ respectively, and a satisfactory spectral purity, with an average phase noise on the order of $-100 \mathrm{dBc} / \mathrm{Hz}$ at $1 \mathrm{MHz}$ offset from the carrier across the $1 \mathrm{GHz}$ tuning bandwidth in (Acampora et al., 2010), allowing for fine spectral resolution. Yet, it suffers from a major drawback, which resides in its tuning function, i.e. the variation of oscillation frequency

${ }^{3}$ This is not the only option. In the case of digital IC for example, the VCOs are based on relaxation oscillators (ring oscillators, delay line oscillators, rotary travelling wave oscillators) which using logical gates synthesize square, triangular, sawtooth waveforms as for example in (Zhou et al., 2011). 
with respect to the control voltages, which in the case of large signal operation sensibly deviates from linear analysis prediction. In (Divina \& Ŝkvor 1998), small signal analysis techniques were used to model the DVCO behaviour, explaining the basic mechanism for which tuning is made possible, which consists in opportunely altering the phase characteristic of the DVCO by changing the transconductance of the active devices through their gate bias voltages. This approach, although analytical, it is limited in that it doesn't allow one to identify important oscillator figures of merit (e.g. oscillation power level, higher order harmonics content, and oscillation's stability) since it only detects the frequency at which oscillations build-up. In order to cope with these issues, nonlinear simulation techniques must be employed in the Time Domain (TD) (Silverberg. \& Wing, 1968; Sobhy \& Jastrzebski 1985) in the Frequency Domain (FD) (Rizzoli et al., 1992) or in a "mixed" Time-Frequency domain (Ngoya \& Larcheveque, 1996). In TD simulations, the differential system of equation is numerically integrated with respect to the time variable, delivering the most accurate representation of the solution waveform, which enables the transient ${ }^{4}$ and the steady state analysis as well. In FD simulations the circuit variables are conveniently expressed in terms of generalized Fourier series ${ }^{5}$, which permits to quickly have information about the steady state, skipping the transient evaluation (Kundert et al., 1990). Application of this principle in microwave circuit analysis gave rise to the Harmonic Balance (HB) method (Rizzoli \& Neri, 1988; Rizzoli et al., 1992) and its extension to modulated signals, the Envelope Transient simulation (Brachtendorf et al., 1998; Ngoya et al., 1995, Ngoya \& Larcheveque, 1996) which is a mixed TD/FD method.

The authors in (Divina \& Škvor, 1998) make use of TD simulations to assess the nonlinear oscillator performance. However, oscillator transient simulations are very time-consuming since many cycles of an high frequency carrier have to be waited out, until the transient is extinguished and the steady state is settled down (Giannini \& Leuzzi, 2004). Furthermore, in the case of the DVCO, transient simulations are often prone to numerical instabilities and convergence failure due to the time domain evaluation of distributed elements which are frequency dispersive (Suarez \& Quèrè, 2003). When analyzing a multi-resonant distributed microwave circuit, with multiple oscillation modes like the DVCO (Acampora et al., 2010; Collado et al., 2010) this issue turns out to be particularly undesireable.

For the aforementioned reasons, in this work the reverse mode DVCO tuning function is calculated by employing HB simulation techniques, opportunely modified to take into account the autonomous nature of the circuit being studied. In fact, an HB simulation of an oscillator circuit is prone to errors like convergence failure or convergence to DC equilibrium point ("zero frequency solution") since it is not externally driven by timevarying RF generators (Chang et al., 1991). Probe methods aim at eliminating the ambiguity by having a fictitious voltage sine-wave RF generator with unknown amplitude and

\footnotetext{
4 This is the reason why often the terms "Time Domain simulation" and "Transient simulation" are often used interchangeably.

${ }^{5}$ Assuming a periodic or quasi-periodic solution exists, it will retain all the features of the RF generators acting as sources. In particular, if $\left(\omega_{1}, \omega_{2}, \ldots, \omega_{k}, \ldots \omega_{n}\right)$ are the $n$ input incommensurable frequencies of the sources, a general circuit variable will contain intermodulation products $\left(p_{1} \omega_{1}+p_{2} \omega_{2}+\ldots+p_{k} \omega_{k}+\ldots+p_{n} \omega_{n}\right)$ where $p_{i}$ are integer coefficients. See (Kundert, 1997, 1999) for more details.
} 
frequency (its phase is conveniently set to zero) inserted at an appropriate node in the circuit in order to force the HB simulator to converge to the oscillating solution. Both amplitude and probe's frequency represent two extra variables which are found by imposing a nonperturbation condition at the node in the circuit to which the probe is connected.

Using these techniques, a reverse mode DVCO has been successfully designed and implemented using standard prototyping techniques and off-the-shelf inexpensive components. The topology of the DVCO resembled a feedack distributed amplifier having four sections and employing a NE 3509M04 HJ-FETs as active elements providing the necessary gain for triggering oscillations. The inter-sections coupling network consited in $\Pi-$ type m-derived sections which comprised lumped inductors and capacitor, the input/output parasitic capacitance of each FET and microstrip line sections providing interconnections and access to each device from the drain line/gate line, and behaved as a low pass structure (Wong, 1993), with a nominal impedance of $50 \Omega$ and a cutoff frequency of $3 \mathrm{GHz}$. Experimental plots revealed a reduction in the frequency tuning range $(0.75-1.85$ $\mathrm{GHz}$ ) with respect to the simulated one $(1-2.4 \mathrm{GHz})$, but still assuring a wideband operation (delivering an $85 \%$ relative tuning range). Phase noise measurements were performed to validate the effectiveness of the proposed DVCO for practical purposes, obtaining a mean value of $-111.2 \mathrm{dBc} / \mathrm{Hz}$ at $1 \mathrm{MHz}$ offset from the carrier, across the overall tuning range. Measured Output Power level was comprised between $+5 \mathrm{dBm}$ and $+7.5 \mathrm{dBm}$.

The chapter is organized as follows. In section 2 the distributed amplifier/oscillator/ VCO working principle is introduced and some examples of its implementation will be given. In section 3 the necessary background in TD/FD simulation techniques is provided with particular emphasis to HB balance/ probe methods for oscillator analysis. Section 4 deals with the analysis and design of a four-section distributed voltage controlled oscillator. Section 5 is devoted to the implementation details and measurements. Last section concludes the work, and paves the way for future research.

\section{Distributed voltage controlled oscillator linear analysis}

This section is aimed at understanding the working principle of Distributed Microwave Amplifiers and Oscillators/VCO.

\subsection{Introduction - Distributed amplifier and oscillator}

In recent years, renewed interest towards distributed microwave circuits has been shown. New architectures for mixers (Safarian et al., 2005), Low Noise Amplifiers (LNA) (Heydary, 2005) oscillators and VCO (Divina \& Škvor, 1998; Wu \& Hajimiri, 2001), have been proposed, and all of them are susceptible to be implemented in integrated form.

Although highly appreciated today, all these circuits share an old discovery patented by Percival in 1937 (Percival, 1937) and later on published by Gintzon (Gintzon et al., 1948) called "distributed amplification". In his work was explained for the first time how to design a very wideband amplifier provided that several active devices should be used. It turned out that the utilization of a pair artificial k-constant transmission line periodically coupled by the active devices' transconductance (Wong, 1993; Pozar, 2004) provided to the overall structure a linear increase in gain and a very wideband operation. The rationale 
behind this improvement lied in the fact that artificial transmission line (ATL) sections, made out of lumped inductances and capacitances, were valuable in diminishing the values of parasitic capacitance seen at the output of a single stage, improving noticeably the bandwidth performance.

In Fig. 1 is depicted a three section distributed amplifier. The signal is injected through the gate line (input ATL) and as the travelling waves pass through each section, it gets amplified at the drain line (output ATL) in a concurrent way. At the end of the gate and at the beginning of the drain line a matched termination section (indicated as a resistor), having the same impedance of the ATL is introduced with the purpose of absorbing the forward propagating waves in the gate line and the backward propagating waves in the drain line (Pozar, 2004). A broadband impedance matching network is placed halfway among the last sections to adjust the impedance levels in order to avoid signal reflections. As the operating frequency increases, the lumped elements should be substituted by properly designed transmission line sections. From this arrangement, a distributed oscillator can be obtained introducing a feedback loop between the output line and the input line of the distributed amplifier (Fig. 2). This topology is known as forward gain distributed oscillator, since it involves forward propagating waves that, circulating in the feedback loop, are re-inserted in the input line through the output node. The feedback path length determines the operating frequency; as the path length gets smaller, the maximum attainable frequency increases. Restricted tuning capabilities can be incorporated in this circuit introducing a varactor diode in the feedback line in order to control its electrical length changing the capacitive loading (Yuen \& Tsang, 2004), or by adequately modifying the bias currents of the active devices to provide "current-steering delay balanced" tuning in (Wu \& Hajimiri, 2001).

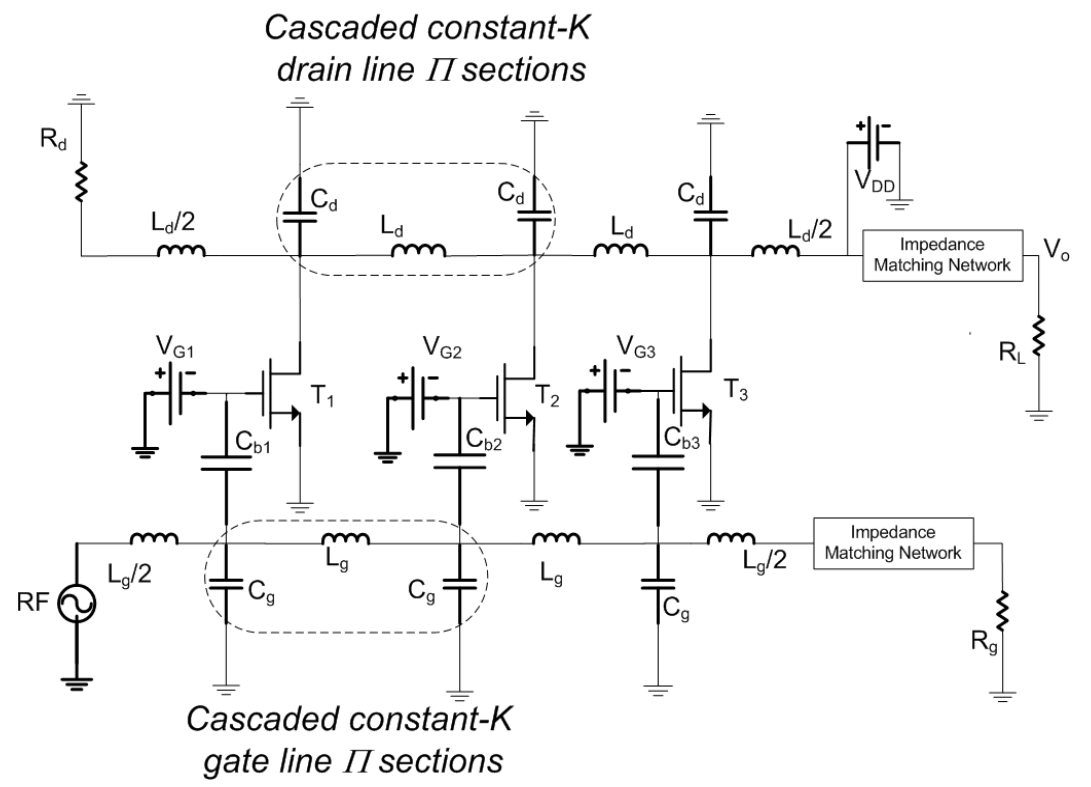

Fig. 1. A three sections distributed amplifier using FETs and Artificial Transmission Lines. 


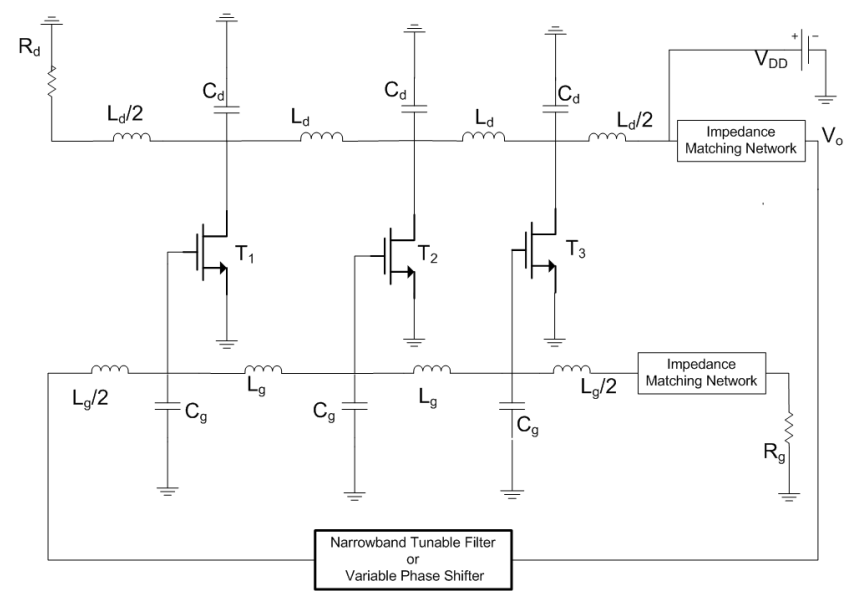

Fig. 2. An ideal DVCO, with a tuning element in the feedback loop.

\subsection{Reverse gain mode distributed voltage controlled oscillator}

An alternative topology for the distributed oscillator was proposed by Škvor (Škvor et al., 1992). The possibility of removing the dummy drain resistor and connecting together the drain and the gate lines in a "reverse manner" was contemplated (Fig. 3) in order to exploit the "backward" scattered waves in the drain line, making them available once more through a feedback loop to the gate line. Compared to the high frequency DVCO proposed in (Wu \& Hajimiri, 2001) it offers several advantages, mainly in terms of greater output power and wider tuning bandwidth (Divina \& Škvor, 1998), at the expense of added complexity residing in the tuning algorithm, which should be fully analyzed employing nonlinear numerical techniques.

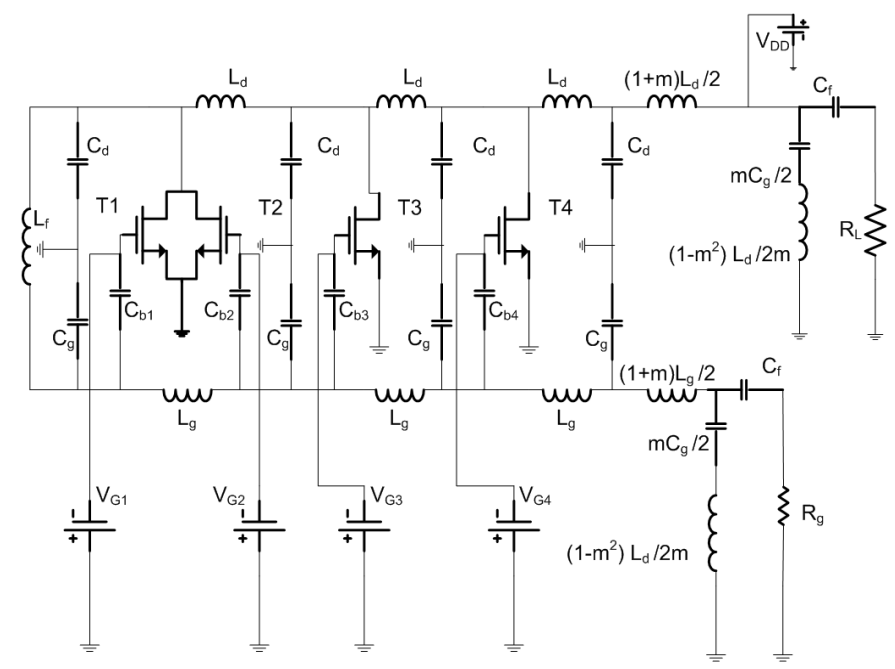

Fig. 3. Idealized Schematic of the Reverse Gain DVCO. 
In this circuit, the effective feedback path length seen by the microwave signals can be modified by activating only one active device at a time, leaving the others switched off. As a consequence, considering a DVCO with $N$ sections, a set of $N$ discrete oscillations will be produced, whose frequencies are distributed within the pass-band of the ATLs (constant kfilter sections (Divina \& Škvor, 1998)). These frequencies have a precise relationship with the cut-off frequency of the $L C$ cells in such a way that the highest frequency component will correspond to the activation of the first transistor, and the lowest frequency will be obtained with the activation of the last active device (Fig.3). Oscillation Frequencies are seen in decreasing order as we subsequently activate each stage, from the first to the last. To estimate them analytically, Barckhausen-Nyquist criteria can be applied in the first place so to find the frequency at which the closed loop gain transfer function equals one, permitting signal regeneration ${ }^{6}$. When the $p$-th stage is activated, oscillation start-up depends on the ratio between the input and the output voltage wave at the $p$-th stage ${ }^{7}$ (called reverse gain) which in turn is influenced by the artificial transmission line impedance $Z^{\pi}(\omega)$, the transconductance of the device itself $g_{m(p)}$, and the phase of the signal across the path from the drain line back to the gate line $\Phi_{r e v}(p)(\omega)$ (Divina \& Škvor, 1995):

$$
G_{r e v}^{(p)}(\omega)=\frac{g_{m(p)} Z_{F}(\omega)}{2} e^{-j\left[\pi+(2 p-1) \psi_{F}(\omega)\right]}=\left|G_{r e v}^{(p)}\right|(\omega) e^{j \Phi_{r e v}^{(p)}(\omega)}
$$

being:

$$
Z^{\pi}(\omega)=Z_{F}(\omega) e^{j \psi_{F}(\omega)}
$$

the impedance of the ח-type LC sections. Applying Nyquist Criterion for the onset of oscillations to $\mathrm{G}_{r e v}(p)(\omega)$, a relation that express the possible self resonant frequencies as a function of the active device position $p$ is obtained (Škvor et al., 1992):

$$
\frac{f^{(p)}}{f_{c}}=\sin \left(\frac{\pi}{4 p-2}\right)
$$

where $f_{c}$ is the cut-off frequency of the low pass structures.

\begin{tabular}{|c|c|}
\hline Device Switched ON $(p)$ & $\begin{array}{c}\text { Oscillation frequencies } \\
\text { (Analytical linear model) }\end{array}$ \\
\hline $\mathrm{T}_{1}$ & $4 \mathrm{GHz}$ \\
\hline $\mathrm{T}_{2}$ & $2 \mathrm{GHz}$ \\
\hline $\mathrm{T}_{3}$ & $1.24 \mathrm{GHz}$ \\
\hline $\mathrm{T}_{4}$ & $880 \mathrm{MHz}$ \\
\hline
\end{tabular}

Table 1. DVCO frequencies for an embedded ATL cut-off frequency of $4 \mathrm{GHz}$.

\footnotetext{
${ }^{6}$ Barckhausen-Nyquist criteria states that for a feedback amplifier with a gain $A(\omega)$ and a feedback transfer function $\beta(j \omega)$, an oscillation occurs at the frequency such that the closed $\left|A\left(\omega_{0}\right) \beta\left(j \omega_{0}\right)\right|=1$, $\measuredangle\left(A\left(\omega_{0}\right) \beta\left(j \omega_{0}\right)\right)=0$.

${ }^{7}$ In the case of simplified linear (small signal) analysis, in which are neglected all the parasitics of the transistors that are simply modeled as controlled current sources, and it is assumed to use the same values for the lumped elements both in the drain an in the gate line. Frequency dependent part only accounts for the impedance of the constant $\mathrm{k}$ - sections.
} 
As an example, in a four sections DVCO with a specified cut-off frequency of $4 \mathrm{GHz}$, the (3) provides the frequencies given in Tab. 1 .

Apart from generating discrete signals, this circuit possess appealing tuning capabilities. In fact, if two stages are activated at the same time, by proper regulation of the biasing voltages $^{8}$ it is possible to get a whole range of frequencies which fall in between the two discrete frequencies related to the activation of each single active device. Its operation principle could be explored analytically, by considering the reverse gain of $p$-th and of $q$-th $(q>p)$ stages (Divina \& Škvor, 1995) when both are simultaneously active:

$$
\begin{aligned}
& G_{r e v}^{(p, q)}(\omega)=\sqrt{\left|G_{r e v}^{(p)}(\omega)\right|^{2}+2\left|G_{r e v}^{(p)}(\omega)\right|\left|G_{r e v}^{(q)}(\omega)\right| \cos \left(\phi_{p}-\phi_{q}\right)+\left|G_{r e v}^{(q)}(\omega)\right|^{2}} \\
& \tan \left(\Delta \Phi_{r e v}^{(p, q)}(\omega)\right)=\frac{\left|G_{r e v}^{(p)}(\omega)\right| \sin \left(\phi_{p}\right)+\left|G_{r e v}^{(q)}(\omega)\right| \sin \left(\phi_{q}\right)}{\left|G_{r e v}^{(p)}(\omega)\right| \cos \left(\phi_{p}\right)+\left|G_{r e v}^{(q)}(\omega)\right| \cos \left(\phi_{q}\right)}
\end{aligned}
$$

Introducing the expression for the reverse gains (5) and simplifying the expression for the phase in (4) one gets:

$$
\begin{gathered}
G_{r e v}^{(p)}(\omega)=\frac{g_{m(p)} Z^{\pi}(\omega)}{2} e^{-j\left[\pi+(2 p-1) \psi_{F}(\omega)\right]} \\
G_{r e v}^{(q)}(\omega)=\frac{g_{m(q)} Z^{\pi}(\omega)}{2} e^{-j\left[\pi+(2 q-1) \psi_{F}(\omega)\right]} \\
G_{r e v}^{(p, q)}(\omega)=\frac{Z^{\pi}(\omega)}{2} \sqrt{g_{m(p)}^{2}+2 g_{m(p)} \cdot g_{m(q)} \cos \left[(q-p) \psi_{F}(\omega)\right]+g_{m(q)}^{2}} \\
\Delta \Phi_{r e v}^{(p, q)}(\omega)=-\frac{\left[\pi+(2 p-1) \psi_{F}(\omega)\right] g_{m p}+\left[\pi+(2 q-1) \psi_{F}(\omega)\right] g_{m q}}{g_{m p}+g_{m q}}
\end{gathered}
$$

which illustrate that the phase of the reverse gain can be changed by means of the transconductance of the active devices $p$ and $q$. This phase variation is the responsible for the frequency tuning in the range $\left[f_{q}, f_{p}\right]$. The best frequency tuning strategy turns out to be based on the complementary biasing of two adjacent active devices $(q=p+1)$. The gate voltage of the $p$-th section is decreased while simultaneously the gate voltage of the $(p+1)$-th section is increased, in order to tune the frequency from $f_{p}$ down to $f_{p+1}$. This process is applied between each pair of transistors to get a tuning range from $f_{N}$ to $f_{1}$ for a DVCO with $N$ sections. In (Škvor et al., 1992) it has been shown that it is necessary to insert an additional transistor, placed "crosswise" between the first and the second active stages, in order to provide a supplementary trans-conductance and consequently achieve a continuous phase variation, assuring smooth tuning. Moreover possible spurious oscillations are avoided by matching terminal sections effectively (with a gate/drain line reflection coefficient not greater than $-20 \mathrm{~dB}$ ideally) to the gate and to the output loads (Divina \& Škvor, 1995) which

8In a common source amplifier, we could vary the input voltage in the gate or the output voltage in the drain to alter the bias of the active devices. The former way is preferred, so when we mention "tuning voltages" or "tuning controls" we will refer to the variation of the gate voltages of the FET devices. 
is accomplished placing an $m$-derived section, just before the drain/gate output terminations. For a value of $m=0.6$ a broadband matching is assured over nearly the $85 \%$ of the band of interest (Wong, 1993).

\subsection{Linear analysis of the DVCO and preliminary design}

A four section reverse mode DVCO can be designed starting from the linear analysis that has been hitherto shown (Acampora et al., 2010; Collado et al., 2010). A simplified DVCO schematic is set up in a commercial microwave circuits' simulator and contains ideal DC blocks and DC feeding networks and resistors in the drain, which are deemed necessary for proper biasing in the real case aren't optimized. In this idealized situation the values of inductances and capacitances chosen for the artificial transmission line sections, are such that the cut-off frequencies both in the gate and in the drain line were of $3 \mathrm{GHz}\left(f_{c}(g)=f_{c}(d)\right)$ and the characteristic impedance $50 \Omega$ providing: $L_{g}\left(L_{d}\right)=5.3 \mathrm{nH}$ and $C_{g}\left(C_{d}\right)=2.1 \mathrm{pF}$. Subsequently, are introduced the four HJ-FET nonlinear models for the NE 3509M04. Each section is analysed with the help of S-parameters, in order to have to have minimum phase mismatch between gate/drain line signal propagation (phase balance). To that aim, several design iteration are necessary to optimize the values of inductances and capacitance to use. Finally, the ideal components are substituted by real ones, introducing the layout elements (microstrips lines, cross, bends and tees), the vendor models for the capacitances and inductances, the interconnecting pads and modelling the parasitics due to the insertion of via holes. Taking into account the new layout constraints the values of the lumped components might be re-tuned several times. In (Acampora et al., 2010) the values $L_{g}\left(L_{d}\right)=3$ $\mathrm{nH}$ and $C_{g}\left(C_{d}\right)=1.2 \mathrm{pF}$ were eventually chosen, providing a cutoff frequency of $5 \mathrm{GHz}$ and a $50 \Omega$ impedance for the basic artificial transmission line sections; the matching $m$-derived sections are then designed accordingly ${ }^{9}$.

In order to get an estimate of the potential oscillation frequencies a preliminary linear analysis is performed. To that aim, the small signal admittance $Y_{p}(f)=G_{p}(f)+j B_{p}(f)=$ $\operatorname{Re}\left(Y_{p}(f)\right)+j \operatorname{Im}\left(Y_{p}(f)\right)$ at a convenient node $P$ in the circuit needs to be evaluated and, in order to have a DC unstable solution (Giannini \& Leuzzi, 2004) frequency regions for which hold $G_{p}(f)<0 ; \partial B_{p}(f) / \partial f>0$ should be sought for different values of the bias voltages. To that aim a small signal voltage probe ${ }^{10}$ is introduced at the node $P$ and a frequency swept AC analysis is carried out, measuring the real and imaginary part admittance function $Y_{p}\left(f, V_{b}, \xi_{1}, \ldots, \xi_{k}\right)=I_{p} / V_{p}$, where $V_{b}$ represent the bias voltage and $\left(\xi_{1}, \ldots, \xi_{k}\right)$ a set of adjustable parameters which can be varied to meet the specifications. The graphs are then displayed and by visual inspection are found the regions in which the admittance real part (conductance) becomes negative presenting "valleys" and its imaginary part (susceptance) presents positive slope. These frequency zones represent unstable DC solution points at

\footnotetext{
${ }^{9}$ The design of the $k$ - constant $L C$ section is performed, via the formulas $Z_{0}=\sqrt{ }(L / C), \omega_{c}=(2 / \sqrt{ }(L C))$ so once the nominal impedance and the cut-off frequency are chosen, $L$ and $C$ are uniquely determined. The $m$ derived section inductance and capacitance values, are related to those used in the $k$-constant LC section, having them multiplied by a scale factor. For $m=0.6$, those values are $C_{m}=(3 / 10) C, L_{m}=(3 / 10) L, \quad L_{p}=(8 / 15) L$, see (Wong, 1993; Pozar, 2004).

${ }^{10 I f}$ the probing voltage needs to cause only slight variation around the transistors' quiescient point, a peak value of $10 \mathrm{mV}$ can be considered "small“when the bias voltages are on the order of 1.5-2V.
} 
which the circuit will likely oscillate. When the two conditions stated above hold, a good estimate of the oscillation frequencies is given by the susceptance intercept of the frequency axis, i.e. the points at which $B_{p}(f)=0$. This linear analysis has shown that potential oscillations could occur along the complete desired frequency band. By turning separately each active device on, a set of four discrete oscillations is obtained, whose estimated frequencies fall within the band [1.2 - 2.6 GHz]. Tuning is achieved continuously since negative conductance zone overlap themselves (Acampora et al., 2010, Collado et al., 2010). However, the linear analysis doesn't provide any information about the steady state oscillation power since it only estimates its frequency, and this approximation could be poor cause of nonlinear operation mode.

\section{Nonlinear simulation techniques for microwave oscillators}

DVCOs have traditionally been studied and designed using linear design tools (Divina \& Škvor, 1998; Wu \& Hajimiri, 2001). However, in order to have a realistic picture that could take into account possible instabilities, waveform distortion, optimal power design, phase noise analysis, harmonic content and other relevant performance parameters, one should have recourse to nonlinear simulation techniques (Kundert, 1999). Taking advantage of the latter it is possible to obtain the DVCO tuning curves, which express the values of the gate voltages necessary to synthesize each of the desired frequencies in a prescribed range, the DVCO power level in across the tuning range and the harmonic rejection (Acampora et al., 2010).

The DVCO behaviour could be analyzed numerically integrating the system of differential equations governing the circuit directly in the time domain (Sobhy \& Jastrzebski, 1985), having a complete representation of the solution throughout an observation range. Nevertheless, this approach presents severe drawbacks in the case of an oscillator for its long computation times, since many periods of a high frequency carrier need to be evaluated until the steady state is finally reached (Giannini \& Leuzzi, 2004). In case of employing microwave distributed elements like in the case of the DVCO, additional processing power is required for representing them in the time domain for they are frequency dispersive; numerical formulation is thus complicated by the introduction of a convolution integral for taking into account this effect. In a DVCO a time domain analysis might be a very frustrating task, considering the possibility of having multi-mode multiple oscillation frequencies which should be manually checked for every particular bias configuration.

A different approach consists in avoid solving the equations in the time domain, but rather use a Fourier series expansion which allow one to transform the original problem into a simpler one, in the frequency domain. This way the entire network is subdivided into two parts; one containing linear microwave devices (both lumped and distributed) which are simply depicted in frequency domain by means of their transfer functions ${ }^{11}$, the other containing nonlinearities for which the time domain description is kept, and Discrete Fourier Transform algorithms are used to switch from one domain to the other. A current

\footnotetext{
${ }^{11}$ Instead of evaluating numerically a complicated convolution integral in time domain, a multiplication of complex quantities is performed, which sensibly relieves the computational load.
} 
balance via the KCL is then established between the two subsets in the form of nonlinear algebraic system of equations involving the unknown coefficients of the Fourier Series Expansion (harmonics), which could be solved by direct or iterative methods (Suarez \& Quéré, 2003). This is the essence of the Piecewise Harmonic Balance (HB), which undoubtedly presents the advantage of detecting the periodic steady state solution avoiding the computation of the initial transients.

\subsection{Harmonic balance for periodically driven microwave circuits}

The starting point for the description of the method (Kundert et al., 1990; Rizzoli et al., 1992; Suarez \& Quéré, 2003) is to split the network in the linear part and in the part containing nonlinear devices; the connection among the two parts is provided at $q$ ports. Nonlinear devices are then represented as nonlinear controlled sources, being dependent upon a set of variables called state variables, which are essential in describing the time evolution of the system. No assumption is made upon the nature of the state variables and on the nonlinearities which can be voltages, currents, fluxes or charges. Lastly, the action of independent sources must be taken into account. A set of three vectors is thus considered, being $\mathbf{x}(t)$ the state variable vector, $\mathbf{y}(t)$ the vector containing the nonlinear controlled sources and the generators $\mathbf{g}(t)$ :

$$
\begin{aligned}
& \mathbf{x}(t)=\left(x_{1}(t), x_{2}(t), \cdots, x_{n}(t)\right)^{\mathbf{T}} \\
& \mathbf{y}(t)=\left(y_{1}(t), y_{2}(t), \cdots, y_{q}(t)\right)^{\mathbf{T}} \\
& \mathbf{g}(t)=\left(g_{1}(t), g_{2}(t), \cdots, g_{s}(t)\right)^{\mathbf{T}}
\end{aligned}
$$

and the most general relationship between $\mathbf{y}(t)$ and $\mathbf{x}(t)$ is of the following form:

$$
\mathbf{y}(t)=\mathbf{\Psi}\left(\mathbf{x}(t), \mathbf{x}(t-\tau), \frac{d \mathbf{x}}{d t}, \cdots, \frac{d^{m} \mathbf{x}}{d t^{m}}\right)
$$

being $\Psi(\cdot)$ a nonlinear function of $\mathbf{x}(t)$, which generally accounts for a dependence upon shifted values of $\mathbf{x}(t)$, and on its derivatives up to $m$-th order. In the following, the case for a single generator (single tone analysis) is described, so the last vector in (7) reduces to a scalar function $g(t)=G_{s} \sin (\omega t)$; generalizations to multi-tone analysis can be found in (Giannini \& Leuzzi, 2004; Suarez \& Quéré, 2003). The second step consists in representing all this variables in a generalized Fourier basis of complex exponentials tones. If the generator drives the circuit with angular frequency $\omega$ :

$$
\mathbf{x}(t)=\sum_{p=-N_{m}}^{N_{m}} \mathbf{x}_{p} e^{j p \omega t}, \mathbf{y}(t)=\sum_{p=-N_{m}}^{N_{m}} \mathbf{Y}_{p} e^{j p \omega t}, g(t)=G_{s} \sin (\omega t)
$$

where only a finite number of harmonics $N_{m}$ has been considered in the expansion. This way the problem is transformed into the frequency domain and the objective becomes the determination of the harmonic components of the two sets of vectors ${ }^{12} \mathbf{X}(\omega), \mathbf{Y}(\omega)$ with

${ }^{12}$ Fourier coefficients of a real valued function possess Hermitian symmetry, i.e. a series coefficient evaluated at a negative index $(-k)$ is the complex conjugate of the same coefficient computed in its 
$\mathbf{X}(\omega)=\left[\mathbf{X}_{-N m}, \ldots, \mathbf{X}_{h}, \ldots, \mathbf{X}_{N m}\right], \quad \mathbf{Y}(\omega)=\left[\mathbf{Y}_{-N m}, \ldots, \mathbf{Y}_{h}, \ldots, \mathbf{Y}_{N m}\right]$ being each column the $h$-harmonic component $\left(-N_{m} \leq h \leq N_{m}\right)$ of the state variables vector and of the nonlinear device outputs. Since nonlinearities don't admit a frequency representation in terms of transfer functions, a Discrete Fourier Transform (indicated with $\mathcal{F}$ ) is employed to toggle from time domain samples to the frequency domain:

$$
\begin{aligned}
\mathbf{X}(\omega) \stackrel{\mathcal{F}^{-1}}{\longrightarrow} \mathbf{x}(t) ; \mathbf{x}(t) \stackrel{\mathbf{\Psi}(\mathbf{x}(t))}{\longrightarrow} \mathbf{y}(t) ; \mathbf{y}(t) \stackrel{\mathcal{F}}{\longrightarrow} \mathbf{Y}(\omega)=\mathcal{F}\left(\mathbf{y}\left(\mathcal{F}^{-1}(\mathbf{X}(\omega))\right)\right) \Rightarrow \\
\mathbf{Y}(\omega)=\mathbf{Y}(\mathbf{X}(\omega))
\end{aligned}
$$

Finally, Kirchhoff Current Law equations are written, balancing the harmonic contributions coming from the vectors $\mathbf{X}(\omega), \mathbf{Y}(\omega)$, and from the driving term:

$$
\mathbf{H}(\mathbf{X}(\omega))=[\mathbf{A}(\omega)] \cdot \mathbf{X}(\omega)-[\mathbf{B}(\omega)] \cdot \mathbf{Y}(\mathbf{X}(\omega))-c \cdot G_{s} \mathbf{e}_{k}=\mathbf{0}
$$

which takes the form of a linear relationship between these three vectors (Rizzoli et al., 1992; Suarez \& Quéré, 2003; Giannini \& Leuzzi , 2004), relating node voltages to branch currents and in which $[\mathbf{A}(\omega)],[\mathbf{B}(\omega)]$ are frequency dependent block diagonal matrix of adequate dimensions, $c$ is a scale factor and $\mathbf{e}_{k}=\left[\delta_{i k}\right]_{1 \leq k \leq n}$ is a $n$ dimensional basis vector. Finally, the nonlinear system of differential equations has been converted to a nonlinear system of algebraic equations (9) which provides an error function. The unknown variables $\mathbf{X}(\omega)$ must satisfy $\mathbf{H}(\mathbf{X}(\omega))=\mathbf{0}$, which can be solved using a multidimensional root finding algorithm like Newton-Raphson (Giannini \& Leuzzi, 2004; Kundert, 1999; Rizzoli et al. 1992; Suarez \& Quéré, 2003). This iterative method, starting from an initial state, iteratively computes the solution by means of a local approximation of the nonlinear function $\mathbf{H}(\cdot)$ to its tangent hyperplane. An outline of the numerical procedure is found in Fig. 4.

For the convergence process to be successful, a good guess of the initial vector $\mathbf{X}_{0}$ is needed, which can be obtained by the (11) under the assumption of low amplitude driving generators, turning off the nonlinearities giving $\mathbf{X}_{0}=c[\mathbf{A}(\omega)]^{-1} G_{s} ; \mathbf{Y}_{0}$ is then derived by means of Fourier Transform pairs like in (10), and a first estimate for $\mathbf{H}(\mathbf{X})$ is built, which will be subsequently corrected. The routine stops when the norm of the error function is less than a certain threshold, which depends on the prescribed accuracy or when the calculated values for the unknown vector $\mathbf{X}$ at two consecutive steps doesn't differ significantly. In terms of computational resources, the heavier step relies on Jacobian matrix computation (by automatic differentiation) and inversion. Therefore different technique could be used, aimed at solving the linearized sytem resulting from a Newton-Raphson iteration with direct methods or with some advanced techniques (Rizzoli et al., 1997).

Compared to Transient/Time Domain Analysis, Harmonic Balance allows evaluating the steady state response of circuits driven by periodic signals in a faster way. However, the assumption upon which the entire Harmonic Balance mathematical framework holds is that the forced circuit will eventually reach its periodic regime, even though in nonlinear

(symmetrical to zero) positive index $(+k)$. Therefore, is possible to set up an Harmonic Balance only for positive frequencies and exploit the last property to compute the coefficients at negative frequencies by a straightforward conjugation, halving the computation time. 
circuits very different long term behaviours are possible ${ }^{13}$ which possibly coexist. Moreover, if the HB algorithm converges, it won't necessarily do to a stable solution that is observable in reality. On the contrary, should the HB algorithm fail to converge, that wouldn't imply there are no stable solutions. Therefore in order to be sure that the mathematical solution matches the actual one could be necessary to undertake further analysis (Giannini \& Leuzzi, 2004).

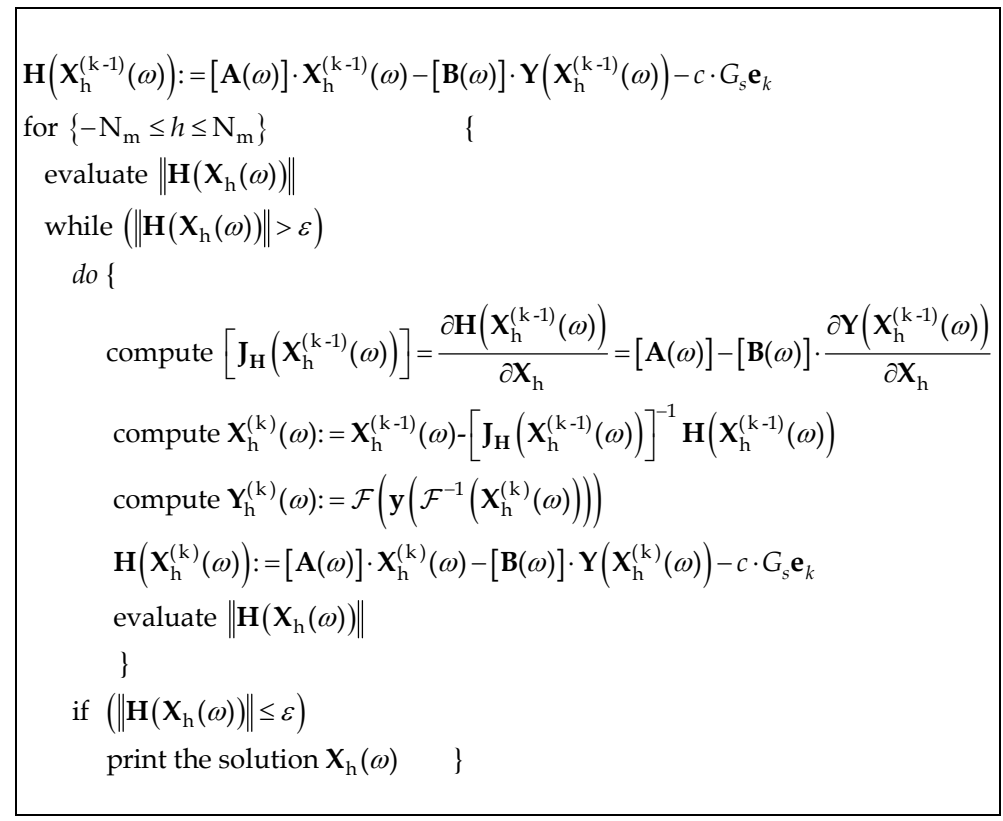

Fig. 4. Numerical Resolution of the Harmonic Balance system of equations.

\subsection{Probe method for oscillator analysis}

Harmonic Balance method proves to be very useful when analyzing circuits that are externally forced by time varying RF generators since they provide a first estimate for the circuit solution. However, convergence problems could result from its use, when dealing with autonomous circuits that present self resonant frequencies or sub-harmonic components, like oscillators, since the actual operating frequency and power of the oscillating solution represent two additional unknowns. Motivated by this lack of knowledge $\mathrm{HB}$ solutions could be misleading; for example in the analysis of a free running oscillator HB method might converge to a degenerate DC steady state solution, as the only generators left are DC sources. To overcome these difficulties, an idealized component called Auxiliary Generator (AG) which fictitiously plays the role of the missing RF generators, is opportunely inserted in our circuit (Giannini \& Leuzzi, 2004; Suarez \& Quéré, 2003) to emulate self resonating frequencies or sub-harmonic components, thus forcing the harmonic balance simulator to find the correct solution. It can be represented by an ideal

${ }^{13}$ Sub-harmonic generation, Chaotic behaviour to cite a few, see (Suarez \& Quéré, 2003). 
sinusoidal voltage source ${ }^{14}$ with series impedance (Thevenin equivalent source) that is being connected in parallel to a circuit node $N$ and defined by its amplitude $\left(A_{p}\right)$ frequency $\left(f_{p}\right)$ and phase $\left(\varphi_{p}\right)$. The series impedance box acts as a very narrowband filter, rejecting all the higher order harmonics while keeping the fundamental ${ }^{15} f_{p}$. Given the time invariance of the solution waveform in a free-running oscillator the phase of the probe is arbitrarily set to zero, while the amplitude and frequency of the oscillation represent two extra-unknowns that augment the dimension of the HB system of equations (HBE) ${ }^{16}$. Therefore two extra equations have to sought, so that the system (11) is not left underdetermined. Since the admittance of the auxiliary generator $Y_{p}$ has to be zero when the circuit is operating in the steady state (as if the probe was left disconnected from the oscillator) in order not to perturb its periodic solution, these equations are chosen to $\operatorname{be}^{17} \operatorname{Re}\left(Y_{p}\left(A_{p}, f_{p}\right)\right)=0, \operatorname{Im}\left(Y_{p}\left(A_{p}, f_{p}\right)\right)=0$. With these added equations the HBE returns square, and a solution can be found both for the oscillation amplitude $A_{0}$ and fundamental frequency $f_{0}$.

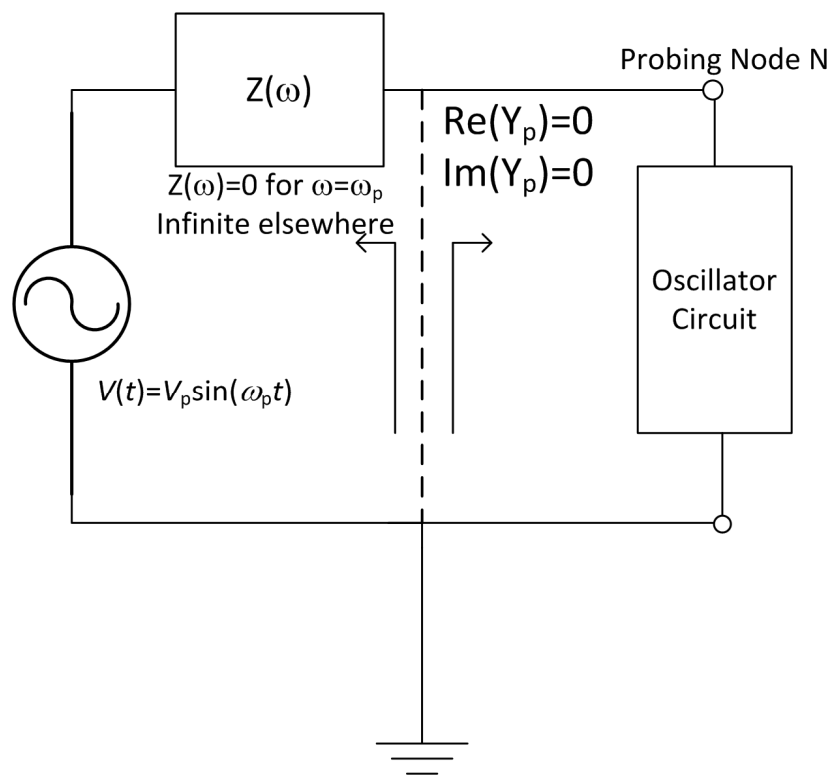

Fig. 5. Auxiliary generator probe.

\footnotetext{
${ }^{14}$ We could also consider an ideal current source with shunt impedance (Norton equivalent source) that is connected in series to a branch of the circuit to be analyzed. In the following, we will employ only AG having voltage sources.

${ }^{15}$ At frequencies other than $f_{p}$ the probe is in fact an open circuit.

${ }^{16}$ In the case of the analysis of a synchronized regime instead, the operation frequency is known (injection frequency) and the variables to be determined are the phase and amplitude of the probe.

${ }^{17}$ In reality, this condition emerge as a particularization of the famous Kurokawa condition for finding large signal steady state oscillation, which states that $Y_{\text {tot }}=Y_{\text {linear }}+Y_{\text {nonlinear }}=0$ at the oscillation frequency where $Y_{\text {linear }}$ is the admittance of the linear subnetwork $Y_{\text {nonlinear }}$ is the admittance of the nonlinear subnetwork, provided the entire network could be partitioned in that way, and the two sub-networks are connected at a single port. The same condition could be written at the probing port providing, the nonperturbation conditions in terms of $Y_{p}$. See (Giannini \& Leuzzi, 2004).
} 
In simulation, these constraints are enforced introducing two optimization goals, both for the real and imaginary parts of the admittance. Then the steady state solution is found running the harmonic balance simulation together with the optimization routine in a two tier scheme; the outer tier is constituted by the optimization algorithm, usually a gradient optimizer, that iteratively computes the candidate solutions $\left(A_{0}, f_{0}\right)$ and pass these values to the inner tier represented by the native $\mathrm{HB}$ algorithm, that solve for all the other circuital variables; if at a given step, the distance between the goals and the computed solution is neglectable according to a predefined metric the solution is found, otherwise the search for the optimal point continues, until the maximum number of iterations is reached. In this optimization method great care has to be taken, with respect to the probe insertion point (Brambilla et al., 2010) and to the probe amplitude and frequency initial estimate. Usually the linear analysis frequency estimate works well for achieving convergence of the HB analysis with the probe method (Chang et al., 1991), selecting randomly the initial values for the AG amplitude. On the contrary, probe amplitude is usually guessed in a trial and error scheme, starting with values that are a tenfold less than the biasing voltages, then trying to increase them as long as convergence failure isn't encountered. A more effective scheme to approach the correct amplitude value, consist in assuming that $\operatorname{Im}\left(Y_{p}(A, f)\right) \sim \operatorname{Im}\left(Y_{p}(f)\right)$ and that $\operatorname{Re}\left(Y_{p}(A, f)\right) \sim \operatorname{Re}\left(Y_{p}(A)\right)$ as for a first order approximation or describing function approach (Giannini and Leuzzi, 2004), in which the susceptance at the node $P$ is mainly a function of the probe frequency and the conductance is mainly a function of the probe amplitude. In this way, having obtained a frequency estimate from initial linear analysis, this can be kept constant while performing a parametric analysis of the circuit w.r.t the parameter $A$, plotting the curve $\operatorname{Re}\left(Y_{p}(A)\right)$ versus $A$, and choosing for $A_{0}$ the value corresponding to the abscissa intercept that fulfil $\operatorname{Re}\left(Y_{p}(A)\right)=0$. Although more complicated, this search method allows one to save many simulation cycles derived by unfruitful attempts.

\section{Harmonic balance DVCO analysis}

In this section Harmonic Balance simulation in a commercial simulator is combined with the use of an auxiliary probe to analyze the DVCO tuning function. Moreover, numerical continuation techniques, used in conjunction with $\mathrm{HB}$ analysis will be employed to show the dependence of the tuning function on some circuit parameters. DVCO nonlinear analysis will thus provide the necessary hints for the synthesis stage.

\subsection{DVCO harmonic balance and parametric analysis}

The DVCO HB analysis starts with the determination of the discrete resonant frequencies (see section 2) obtained by independently biasing each active device. Therefore having chosen the NE3509M04 the biasing voltage of the active device is chosen in the interval $[-0.4 \mathrm{~V}, 0 \mathrm{~V}]$ according to its electrical characteristic. Subsequently four HB simulations are performed, choosing the maximum harmonic order $N_{\max }=3$, and introducing the auxiliary generator in the vicinity of the DVCO feedback loop to find the oscillation frequencies and output power level. As an acceptable approximation for $\operatorname{Re}\left(Y_{p}\right), \operatorname{Im}\left(Y_{p}\right)=0$ could be considered $-1 \cdot 10^{-18}(S) \leq \operatorname{Re}\left(Y_{p}\right)$, $\operatorname{Im}\left(Y_{p}\right) \leq 1 \cdot 10^{-18}(\mathrm{~S})$ which constitute two goal to be fulfilled by the gradient optimizer as detailed in Fig. 6, where the flowchart for finding the DVCO discrete resonant frequencies and the corresponding oscillation power is shown with greater detail. 

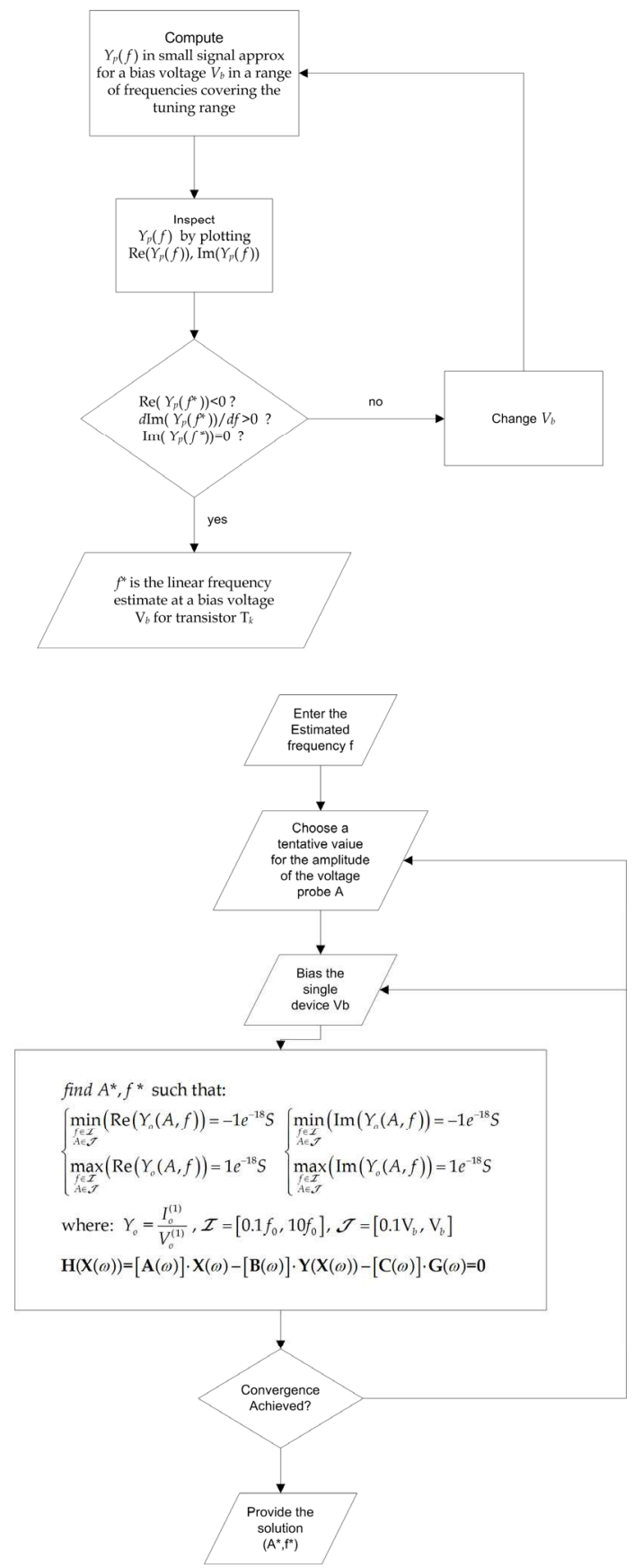

Fig. 6. Double Flowchart for computing the DVCO output spectrum for the discrete oscillation, starting from their small signal frequency estimate. 


\begin{tabular}{|l|c|c|c|}
\hline Active device & Oscillation Frequency & Output power & Biasing Voltage \\
\hline $\mathrm{T}_{1}$ & $2.35023 \mathrm{GHz}$ & $2.682 \mathrm{dBm}$ & $-0,2015 \mathrm{~V}$ \\
\hline $\mathrm{T}_{2}$ & $1.59917 \mathrm{GHz}$ & $1.966 \mathrm{dBm}$ & $-0.2055 \mathrm{~V}$ \\
\hline $\mathrm{T}_{3}$ & $1.13392 \mathrm{GHz}$ & $0.762 \mathrm{dBm}$ & $-0.1000 \mathrm{~V}$ \\
\hline $\mathrm{T}_{4}$ & $0.92538 \mathrm{GHz}$ & $-3.017 \mathrm{dBm}$ & $-0.1414 \mathrm{~V}$ \\
\hline
\end{tabular}

Table 2. Individual Oscillation Frequencies and Power level.

After the discrete resonant frequencies has been obtained, sensitivity with respect to the lumped components is investigated, by means of a parametric analysis (Collado et al., 2010). To that aim, the inductances $\left(L_{g}, L_{d}\right)$ and the capacitances $\left(C_{g}, C_{d}\right)$ are varied in a certain range and the evolution of the oscillatory solutions is traced for each of the four active device separately. Since in our design should be $L_{g}=L_{d}=L, C_{g}=C_{d}=C$, with $1 \mathrm{nH} \leq L \leq 5 \mathrm{nH}$ and $1 \mathrm{pF} \leq C \leq 3 \mathrm{pF}$, the solution is found once the large signal steady state condition $\operatorname{Re}\left(Y_{p}(A, f)\right)=0, \operatorname{Im}\left(Y_{p}(A, f)\right)=0$ and the parameter dependent $\operatorname{HBE} \mathbf{H}(\mathbf{X}, L, C)=\mathbf{0}$ are simultaneously fulfilled. In a commercial simulator, this has been checked running a parameter swept optimization routine. Since there are two parameters, a double sweep is necessary, the first one for changing the capacitance value and the second (nested in the first) for varying the inductance as illustrated in (Collado et al., 2010).

\subsection{DVCO tuning function and stability analysis}

Once it has been checked that each of the transistors lead to oscillations distributed along the frequency band, the tuning capabilities of the circuit are analyzed. A modification of the previous routine is used in that the auxiliary generator frequency $f_{p}$ is increasingly swept in steps $\left(50 \mathrm{MHz}\right.$ is usually enough to ensure convergence) and the gate voltages $V_{g}(i), V_{g}(j)$ ( $j=i+1, i=1,2,3)$ necessary to synthesize each of the frequencies in the sweep are calculated as additional optimization variables that fulfil the non perturbation conditions $\operatorname{Re}\left(Y_{p}\left(f_{p}, A_{p}\right.\right.$, $\left.\left.V_{g}(i), V_{g}(j)\right)\right)=0, \operatorname{Im}\left(Y_{p}\left(f_{p}, A_{p}, V_{g}(i), V_{g}(j)\right)\right)=0$. In order to obtain the tuning characteristic of the circuit, the frequency tuning band has been divided in three "zones". In each of the zones only two gate voltages are modified to achieve oscillator tuning according to (Divina \& Škvor, 1998) while the rest of the transistors are deactivated at $V_{g}(o f f)=-1 V$. From an empirical point of view, is noticed that the convergence of the swept optimization process depends, as in the previous case, upon a suitable selection of the initial candidates value for $A_{p}, V_{g}(i), V_{g}(j)$. The tuning voltages of the active sections are initially chosen to be equal to the midpoint of the voltage range in which both devices' transconductance is nonzero, which corresponds to $\mathrm{V}_{g}(o n)=-0.3 \mathrm{~V}$. Subsequently a single frequency point optimization is performed, in order to obtain the values of the oscillation amplitude and frequency for that particular bias configuration. Finally, the frequency swept optimization curves routine starts as described earlier. In case convergence failure should occur, one has to re-initialize $A_{p}$, $V_{g}(i), V_{g}(j)$ and attempt sweeping the frequency decreasingly. If neither this helps in reaching a solution, numerical continuation techniques have to be invoked. 


\begin{tabular}{|l|l|l|}
\hline Frequency Zone & Active Devices & $\boldsymbol{\Delta} \boldsymbol{f ( G H z )}$ \\
\hline Highest, I & $\mathrm{T}_{1}, \mathrm{~T}_{2}$ & $1.7 \div 2.4$ \\
\hline Central, II & $\mathrm{T}_{2}, \mathrm{~T}_{3}$ & $1.7 \div 1.25$ \\
\hline Lowest, II & $\mathrm{T}_{3}, \mathrm{~T}_{4}$ & $1.0 \div 1.25$ \\
\hline
\end{tabular}

Table 3. Frequency zones and corresponding active devices operating.

The evolution of the gate voltages versus frequency confirms almost perfectly the theoretical predictions (Divina \& Škvor, 1998; Škvor et al., 1992), showing a complementary variation on the tuning voltages in pairs. Besides, the DC current consumption reveals a close relationship with the output power level, showing nearly identical trends. It can be observed in (Fig.7) that the output power has larger variations along the edges of each of the frequency tuning zones. A similar behavior can be observed in the DC current consumption. This phenomenon is comprehensible, if it is taken into account that when approaching the border between the zone $z$-th and $(z+1)$-th, three gate voltages namely $V_{g}(z-1), V_{g}(z), V_{g}(z+1)$, should come into play in determining the oscillation frequency as it is evident from Table 2. In fact, this abrupt edge-variation has been subsequently corrected, enforcing an additional constraint for the output power and using an additional gate voltage as optimization variable. A heuristic approach was adopted to achieve an output power range that ensured less power fluctuations and no discontinuities in the frequency tuning range. The constraint on the output power has been made tighter in steps. Starting from the initial constraint that limited the output power to fall in the range $[-8 \mathrm{dBm}, 8 \mathrm{dBm}]$, this range has been halved in the subsequent steps; having checked the frequency tuning curves to guarantee the circuit did not lose its tuning capabilities. The result of applying this optimization process can be seen in Fig.8. The necessary gate voltages in order to tune the frequency and at the same time maintain the output power characteristic inside the variation limits are represented. The output power variation along the frequency tuning band is shown. After numerous simulations, the output power goal range has been chosen to be $[3 \mathrm{dBm}, 7 \mathrm{dBm}]$, assuring a maximum difference in the output power of $4 \mathrm{~dB}$ for nearly $96 \%$ of the tuning bandwidth.
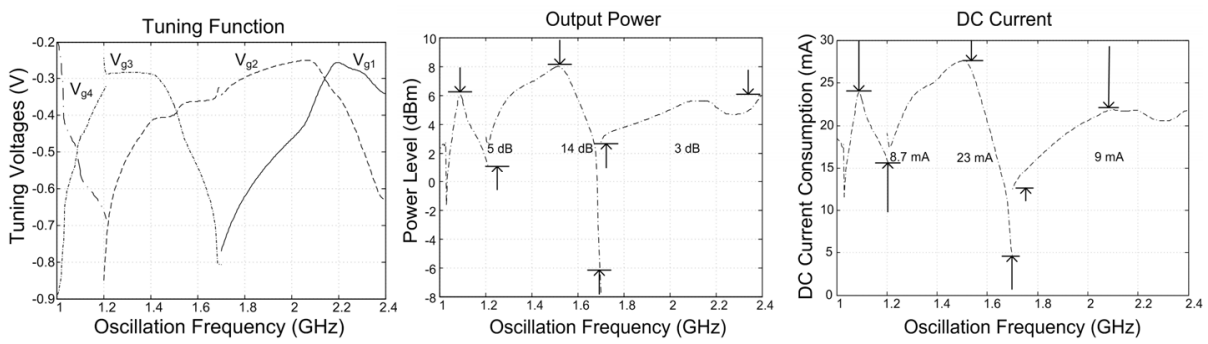

Fig. 7. Tuning Function, Output Power level, DC current for the simulated DVCO. 

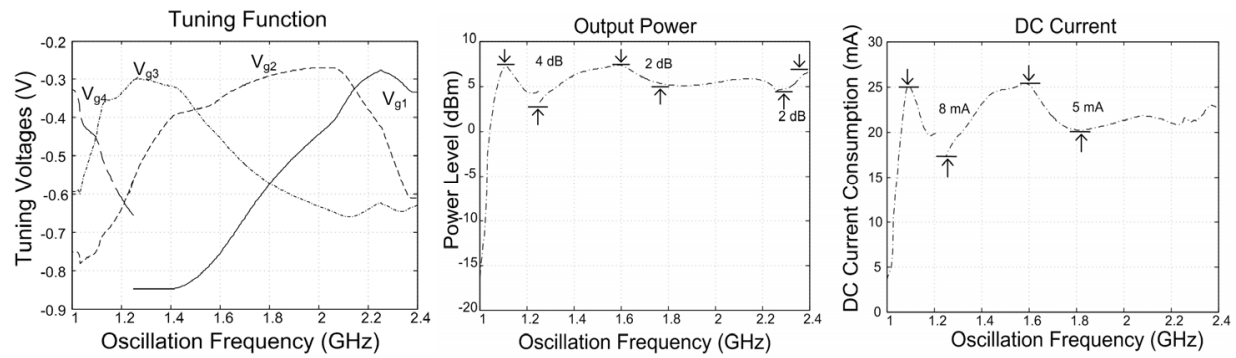

Fig. 8. Tuning range obtained varying the gate voltages, after power optimization.

A power overshoot is still present at the very beginning of the band, between 1 and $1.1 \mathrm{GHz}$ while intermediate power drops have been completely removed. Furthermore in more than $60 \%$ of the tuning band (from $1.35 \mathrm{GHz}$ to $2.4 \mathrm{GHz}$ ) power has shown limited fluctuations: 2 $\mathrm{dB}$ in the frequency span from $1.35 \mathrm{GHz}$ (approx.) to $2.25 \mathrm{GHz}$ (approx.) and the same amount in the interval between 2.25 and $2.4 \mathrm{GHz}$, with an absolute variation of $2.5 \mathrm{~dB}$. Similar remarks are valid for the DC current consumption. A current overshoot takes place at the beginning of the band, while the maximum absolute variation in the dissipated current is $8 \mathrm{~mA}$ and occurs locally (at $1.25 \mathrm{GHz}$ approx). In the rest of the band variations are kept limited to $5 \mathrm{~mA}$.

The assumption made up to now is that if the convergence of the harmonic balance system of equations and the condition at the probe port $\operatorname{Re}\left(Y_{p}\right)=0, \operatorname{Im}\left(Y_{p}\right)=0$ are simultaneously satisfied the solution will be unique. However, due to the particular configuration of the DVCO, multiple oscillating modes are possible, each one characterized by different power level (Acampora et al., 2010). Thus, the solution represented in fig. 9-10 represents in effect one member of a family of solutions whose stability needs to be investigated. In this regard, double parametric sweeps have been performed (Collado et al., 2010) choosing as parameters $\left(V_{g}^{(1)}, V_{g}^{(2)}\right)$ in the first zone $\left(V_{g}(2), V_{g}\left({ }^{(3)}\right)\right.$ in the second zone and $\left(V_{g}(3), V_{g}^{(4)}\right)$ in the third zone and iteratively running $\mathrm{HB}$ simulations for $-1 \mathrm{~V} \leq\left(V_{g}{ }^{(i)}, V_{g}{ }^{(i+1)}\right) \leq 0 \mathrm{~V}$. The curves shown in (Collado et al., 2010) illustrate that it's possible to synthesize the same frequency with more than one bias combination.

\section{DVCO implementation and measurements results}

After the nonlinear simulations were carried out, a DVCO prototype was fabricated taking advantage of local facilities (Acampora et al., 2010; Collado et al., 2010). The layout of the device was realized on a $20 \mathrm{mil}(0.5 \mathrm{~mm})$ thick Arlon A25N substrate, where the microstrip lines' geometries were grooved using a mechanical milling machine. Lumped inductors and capacitors from TOKO were soldered on the circuit, which was tested several times in order to ensure it would comply with the expectations. For the measurement phase, five independent digitally controlled voltage sources were used; four of them were the negative tuning voltages to adjust the oscillation frequency, and the last one was the constant drain line voltage, which was common to all the sections. The circuit is shown in Figure 9. 


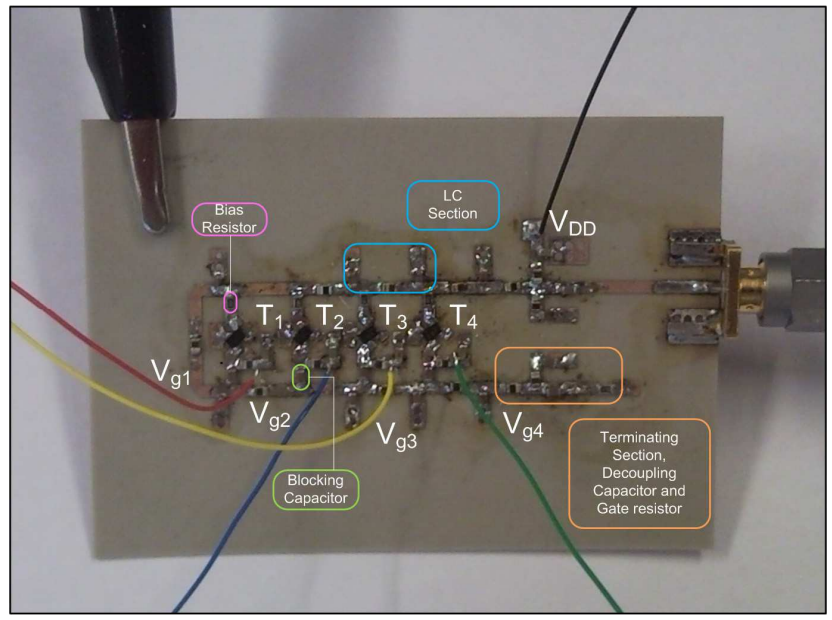

Fig. 9. A prototype of the DVCO.

For keeping track of the oscillation frequency and power an Agilent Spectrum Analyser E4448A (Fig. 10) was used, having an operating bandwidth from $3 \mathrm{GHz}$ to $50 \mathrm{GHz}$. The results are shown in the pictures below (Fig. 11). It is seen that experimental tuning characteristic, power and DC current consumption curves match the theoretical predictions, although the tuning bandwidth is somehow shifted towards lower values. This effect is most likely due to a mismatch between vendor's model and real characteristic for the discrete component and active devices. Moreover, in the schematic used for simulation, coupling effect between adjacent lines has been neglected, and since resistors model from the vendor were unavailable, they have been approximated with ideal elements.

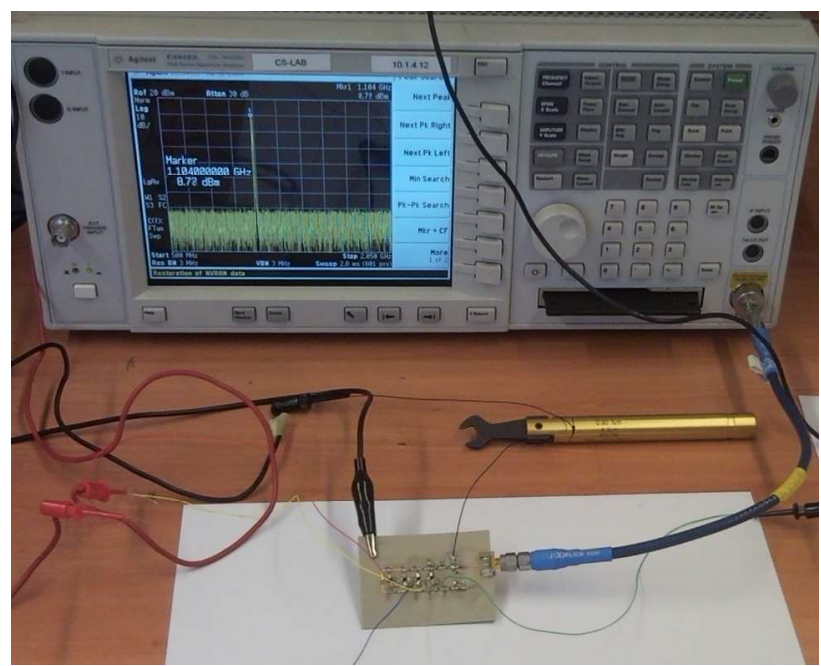

Fig. 10. Measurement benchmark for deriving the tuning curves, output power and DC current for the DVCO. 

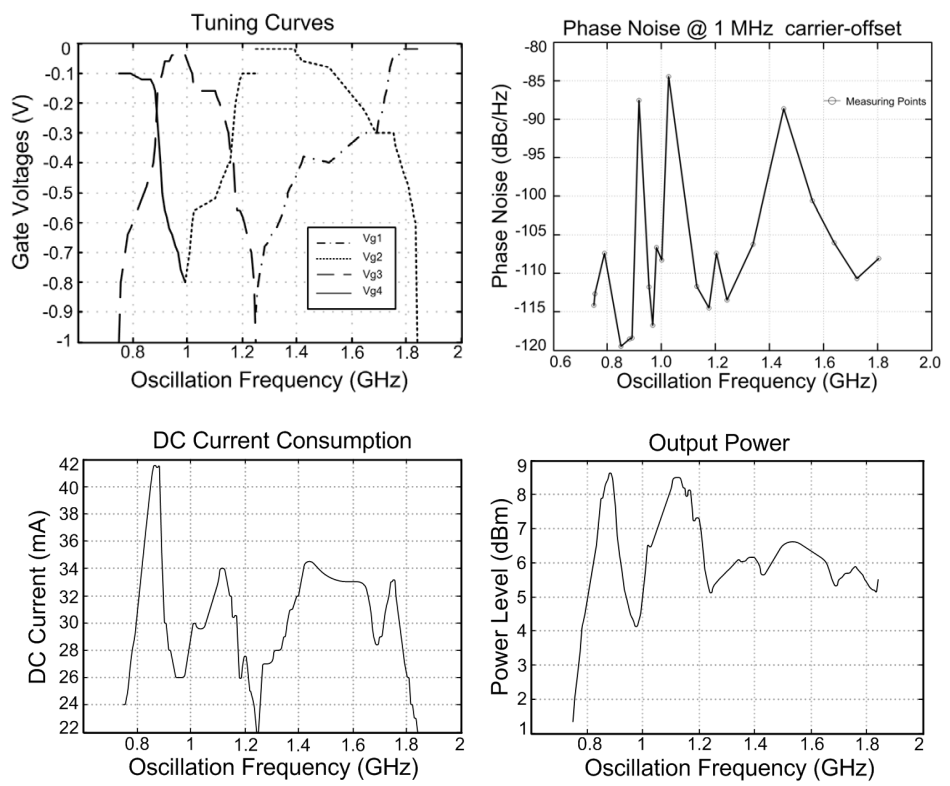

Fig. 11. Measurement results in terms of tuning curves, output power, DC current consumption at phase noise at $1 \mathrm{MHz}$ offset from the carrier, across the tuning band.

In the first prototypes, much attention was devoted to kill some parasitic oscillations that prevented the DVCO from having a continuous tuning function. In these cases, many simulations were made to find out the optimal value of the drain bias resistor that would eliminate the spurious oscillation.

However, still the experimental tuning function suffered from instabilities and hysteresis phenomena. Ambiguous tuning function behaviour has been experimentally substantiated. In fact keeping one voltage fixed at a certain value and changing a neighbouring one, the output oscillation didn't change its frequency but its power. In some cases, oscillations "jumps" in frequency have been noticed. It has been observed empirically that all these issue might be mitigated at least, if the drain voltage $V_{D D}$ is varied in order to keep the DC current $I_{D D}$ constant and to low values (on the order of 10 $\mathrm{mA}$ ). Maintaining $\mathrm{I}_{\mathrm{DD}}$ low, appears to be beneficial also in terms of phase noise, where is seen that poorest phase noise performance corresponds to those oscillations whose DC current consumption is elevated.

\section{Conclusion}

The need for flexible/smart protocols in which radio resources are dynamically allocated among users across a wide frequency band, calls for improvement on communication subsystems employed on user equipments. Since oscillators, VCOs and other oscillatordriven systems are remarkably important elements in every RF front-end; it is believed that they should be endowed with wideband spectrum sensing capability to accommodate the needs of Cognitive Radio technology. 
To that aim, Distributed Voltage Controlled Oscillators have been investigated in this chapter, pointing out their general features and discussing with greater detail one circuit that provides very wideband tuning capabilities namely the "reverse mode DVCO". Starting from consolidated results, our purpose has been to shed new light on DVCO operation with the help of nonlinear analysis and simulation tools, which has allowed to confirm the theoretical predictions already established and to proceed further in investigating the tuning function, the output power variation across the tuning band, and DC current consumption. Moreover, a prototype of the DVCO has been fabricated utilizing discrete components, on a microstrips printed circuit board. The measurements results matched adequately the nonlinear simulations. Nevertheless more research will be needed to discover a suitable tuning algorithm.

Future research tasks include the implementation of a Distributed Divider, and studying the injection locking properties of the DVCO (Paciorek, 1965; Tsang \& Yuen, 2004). Early tests with a slightly modified version of the DVCO shown in this chapter manifested its super harmonic and sub harmonic injection locking properties, allowing the synchronization of the DVCO output with an external signal whose frequency is a multiple (double or triple) with respect to the fundamental in the case of super-harmonic injection locking or a submultiple, (half of the fundamental) in the case of sub-harmonic injection locking.

\section{References}

Acampora, A.; Collado, A. \& Georgiadis, A.(2010). Nonlinear analysis and optimization of a Distributed Voltage Controlled Oscillator for Cognitive Radio, Proceedings of the 2010 IEEE International Microwave Workshop Series (IMWS) on RF Front-ends for Software Defined and Cognitive Radio Solutions, vol., no., February 2010, pp.1-4

Akyildiz, I. F.; Lee, W.; Vuran, M. C. \& Mohanty, S. (2006). NeXt generation/dynamic spectrum access/cognitive radio wireless networks: a survey. Elsevier Journal on Computer Networks, vol. 50, no. 13, May 2006, pp.2127 - 2159

Brachtendorf, H.G.; Welsch, G. \& Laur, R. (1998). A time-frequency algorithm for the simulation of the initial transient response of oscillators. Proceedings of the 1998 IEEE International Symposium on Circuits and Systems, (ISCAS '98) vol.6, no., 31 May-3 Jun 1998, pp.236-239

Brambilla, A.; Gruosso, G.; Gajani, G.S. (2010). Robust Harmonic-Probe Method for the Simulation of Oscillators, IEEE Transactions on Circuits and Systems I: Regular Papers, vol.57, no.9, Sept. 2010, pp.2531-2541

Chang, C.-R.; Steer, M.B.; Martin, S. \& Reese, E. Jr. (1991). Computer-aided analysis of freerunning microwave oscillators, IEEE Transactions on Microwave Theory and Techniques, vol. 39, no. 10, October 1991 pp.1735-1745

Christoffersen, C.E.; Ozkar, M.; Steer, M.B.; Case, M.G. \& Rodwell, M. (1999). State-variablebased transient analysis using convolution. IEEE Transactions on Microwave Theory and Techniques, vol.47, no.6, pp.882-889, June 1999

Cohen, L.D. (1979). Varactor Tuned Gunn Oscillators with Wide Tuning Range for the 25 to $75 \mathrm{GHz}$ Frequency Band. Proceedings of the 1979 IEEE MTT-S International Microwave Symposium Digest, , vol., no., April 30-May 2, 1979, pp.177-179

Collado, A.; Acampora, A. \& Georgiadis, A. (2010). Nonlinear analysis and synthesis of distributed voltage controlled oscillators. International Journal of Microwave and Wireless Technologies, vol.2, no.2, April 2010, pp.159-163 
Divina L. \& Škvor Z. (1995). Experimental verification of a distributed amplifier oscillator, Proceedings of the 25th European Microwave Conference (EuMC) 1995, Kent, U.K. Nexus Media Limited, September 1995, pp. 1163-1167

Divina, L. \& Škvor, Z. (1998). The Distributed Oscillator at 4 GHz. IEEE Transactions On Microwave Theory And Techniques, vol. 46, no. 12, December 1998, pp 2240-2243

Ferrus, R.; Sallent, O. \& Agusti, R. (2010). Interworking in heterogeneous wireless networks: Comprehensive framework and future trends, IEEE Wireless Communications Magazine, vol.17, no.2, April 2010, pp.22-31

Frei, S.; Fuhrmann, W.; Rinkel, A. \& Ghita, B.V. (2011). Improvements to Inter System Handover in the EPC Environment, Proceedings of the 2011 4th IFIP International Conference on New Technologies, Mobility and Security (NTMS), vol., no., February 2011, pp.1-5, 7-10

Giannini F. \& Leuzzi G. (June 28, 2004). Nonlinear Microwave Circuit Design, Wiley, ISBN: 978-0470847015

Ginzton E. L.; Hewlett, W. R.; Jasberg, J. H. \& Noe, J. D. (1948). Distributed Amplification Proceedings of the IRE, August 1948, pp. 956-69

Heydary, P. (2005). Design and Analysis of A Performance-Optimized CMOS UWB Distributed LNA. IEEE Journal Of Solid-State Circuits, vol.42, no.9, September 2007, pp.1892-1905

Kundert K.S. (1999). Introduction to RF simulation and its application, IEEE Journal on SolidState Circuit, 34, September 1999, pp. 1298-1319, 14.09.2011, Available also from: http://www.designers-guide.org/Analysis/rf-sim.pdf (October 2010)

Kundert, K.S. (1997). Simulation methods for RF integrated circuits, Computer-Aided Design, 1997. Digest of Technical Papers., 1997 IEEE/ACM International Conference on, vol., no., November 1997, pp.752-765, 9-13

Kundert, K.S.; White, K.S. \& Sangiovanni-Vincentelli, A. (March 31, 1990). Steady-state methods for simulating analog and microwave circuits, Kluwer Academic Publishers, March 1990, Springer reprint of hardcover 1st edition, December 2010, ISBN: 9781441951212

Ngoya, E. \& Larcheveque, R. (1996). Envelope transient analysis: A new method for the transient and steady state analysis of microwave communication circuits and systems, Proceedings of the IEEE MTT-S International Microwave Symposium Digest, 1996, vol.3, no., June 1996, pp.1365-1368

Ngoya, E.; Suarez A.; Sommet R. \& Quéré, R. (1995). Steady state analysis of free or forced oscillators by harmonic balance and stability investigation of periodic and quasiperiodic regimes. Wiley International Journal on Microwave and Millimeter Wave Computer Aided Engineering, vol. 5, no. 3, May 1995, pp. 210-223

Paciorek, L.J. (1965). Injection locking of oscillators, Proceedings of the IEEE, vol.53, no.11, November 1965, pp. 1723- 1727

Percival, W. S. (1937). Thermionic Valve Circuits. British Patent Specification no. 460,562, filed 24 July 1936, granted January 1937

Peterson, D.F. (1980). Varactor Properties for Wide-Band Linear-Tuning Microwave VCO's. IEEE Transactions on Microwave Theory and Techniques, vol.28, no.2, Februay 1980, pp. 110- 119

Pozar D. (February 5, 2004). Microwave Engineering, Wiley, New York, ISBN: 9780471448785 
Rizzoli, V. \& Neri, A. (1988). State of the art and present trends in nonlinear microwave CAD techniques IEEE Transactions on Microwave Theory and Techniques, vol. 36, February 1988, pp. 343-356

Rizzoli, V.; Lipparini, A.; Constanzo, A.; Mastri, F.; Neri, A. \& Massoti, S. (1992). State of the art harmonic balance simulation of forced nonlinear microwave circuits by the piecewise technique, IEEE Transactions on Microwave Theory and Techniques, vol. 40, no., January 1992, pp. 12-28

Rizzoli, V.; Mastri, F.; Cecchetti, C.; Sgallari, F. (1997). Fast and robust inexact Newton approach to the harmonic-balance analysis of nonlinear microwave circuits, IEEE Microwave and Guided Wave Letters, vol.7, no.10, October 1997, pp.359-361

Safarian, A. Q.; Yazdi, A. \& Yedari, P. (2005). Design and Analysis of an Ultrawide-Band Distributed CMOS Mixer. IEEE Transactions On Very Large Scale of integration (VLSI) Systems, vol.13, no.5, May 2005, pp 618-629

Silverberg, M. \& Wing, O. (1968). Time Domain Computer Solutions for Networks Containing Lumped Nonlinear Elements. IEEE Transactions on Circuit Theory, vol.15, no.3, September 1968 pp. 292- 294

Škvor, Z.; Saunders, S.R. \& Aitchison, C.S. (1992). Novel decade electronically tunable microwave oscillator based on the distributed amplifier. IEEE Electronics Letters, vol.28, no.17, August 1992, pp.1647-1648

Sobhy, M. I. \& Jastrzebski, A. K. (1985). Direct Integration Methods of Non-Linear Microwave Circuits. Proceedings of the 15th European Microwave Conference, 1985, vol., no., September 1985, pp.1110-1118

Suarez, A. \& Quéré, R. (January 2003). Stability Analysis of Nonlinear Microwave Circuits, Artech House publishers, ISBN: 978-1580533034

Sun, Y. (1972). Generation of Sinusoidal Voltage (Current)-Controlled Oscillators for Integrated Circuits. IEEE Transactions on Circuit Theory, vol.19, no.2, March 1972, pp. 137- 141

Tsang, K.F. \& Yuen, C. M. (2004). A 2.7 V, $5.2 \mathrm{GHz}$ frequency synthesizer with 1/2 subharmonically injection-locked distributed voltage controlled oscillator. IEEE Transactions on Consumer Electronics, vol.50, no.4, November 2004, pp. 1237- 1243

Wireless World Research Forum Working Group 6 (2004). Cognitive Radio, Spectrum and Radio Resource Management (White Paper), 14.09.2011, Available from http://www.wireless-world-research.org/fileadmin/sites/default/files/about _the_forum /WG/WG6/White\%20Paper/WG6_WP4.pdf

Wong, T.T.Y. (1993). Fundamentals of Distributed Amplification, 1993, Artech House, London, ISBN: 978-089-0066-15-7

Wu, H. \& Hajimiri, A. (2000). A $10 \mathrm{GHz}$ CMOS distributed voltage controlled oscillator, Proceedings of the IEEE 2000 Custom Integrated Circuits Conference (CICC)., vol., no., May 2000, pp.581-584

Wu, H. \& Hajimiri, A. (2001). Silicon-Based Distributed Voltage-Controlled Oscillators. IEEE Journal Of Solid-State Circuits, vol. 36, no. 3, March 2001, pp 493-402

Yuen, C. M. \& Tsang, K. F. (2004). A 1.8-V distributed voltage-controlled oscillator module for 5.8-GHz ISM band. IEEE Microwave and Wireless Components Letters, vol.14, no.11, November 2004, pp. 525- 527

Zhou, B.; Rhee, W. \& Wang, Z. (2011). Relaxation oscillator with quadrature triangular and square waveform generation, IEEE Electronics Letters, vol.47, no.13, June 2011, pp.779-780 


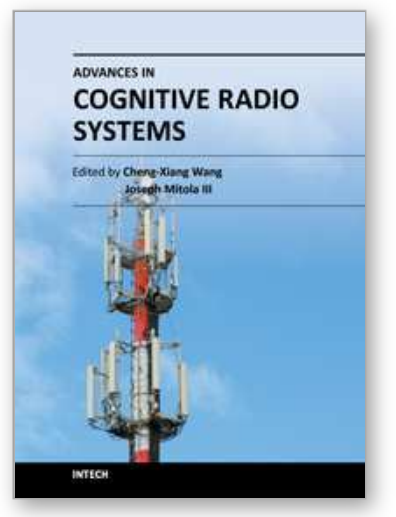

\section{Advances in Cognitive Radio Systems \\ Edited by Dr. Cheng-Xiang Wang}

ISBN 978-953-51-0666-1

Hard cover, 150 pages

Publisher InTech

Published online 05, July, 2012

Published in print edition July, 2012

\section{How to reference}

In order to correctly reference this scholarly work, feel free to copy and paste the following:

Alessandro Acampora and Apostolos Georgiadis (2012). Wideband Voltage Controlled Oscillators for Cognitive Radio Systems, Advances in Cognitive Radio Systems, Dr. Cheng-Xiang Wang (Ed.), ISBN: 978-953-51-06661, InTech, Available from: http://www.intechopen.com/books/advances-in-cognitive-radio-systems/widebandvoltage-controlled-oscillators-for-cognitive-radio-systems

\section{INTECH}

open science | open minds

\section{InTech Europe}

University Campus STeP Ri

Slavka Krautzeka 83/A

51000 Rijeka, Croatia

Phone: +385 (51) 770447

Fax: +385 (51) 686166

www.intechopen.com

\section{InTech China}

Unit 405, Office Block, Hotel Equatorial Shanghai

No.65, Yan An Road (West), Shanghai, 200040, China

中国上海市延安西路65号上海国际贵都大饭店办公楼405单元

Phone: +86-21-62489820

Fax: +86-21-62489821 
(C) 2012 The Author(s). Licensee IntechOpen. This is an open access article distributed under the terms of the Creative Commons Attribution 3.0 License, which permits unrestricted use, distribution, and reproduction in any medium, provided the original work is properly cited. 